\title{
Non-uniform liquid-crystalline phases of parallel hard rod-shaped particles: From ellipsoids to cylinders.
}

\author{
Y. Martínez-Ratón ${ }^{\circledast}$ \\ Grupo Interdisciplinar de Sistemas Complejos (GISC), Departamento de Matemáticas, \\ Escuela Politécnica Superior, Universidad Carlos III de Madrid, \\ Avenida de la Universidad 30, E-28911, Leganés, Madrid, Spain \\ E. Velascd \\ Departamento de Física Téorica de la Materia Condensada and Instituto de Ciencia de Materiales Nicolás Cabrera, \\ Universidad Autónoma de Madrid, E-28049 Madrid, Spain
}

(Dated: February 26, 2008)

\begin{abstract}
In this article we consider systems of parallel hard superellipsoids, which can be viewed as a possible interpolation between ellipsoids of revolution and cylinders. Superellipsoids are characterized by an aspect ratio and an exponent $\alpha$ (shape parameter) which takes care of the geometry, with $\alpha=1$ corresponding to ellipsoids of revolution, while $\alpha=\infty$ is the limit of cylinders. It is well known that, while hard parallel cylinders exhibit nematic, smectic, and solid phases, hard parallel ellipsoids do not stabilize the smectic phase, the nematic phase transforming directly into a solid as density is increased. We use computer simulation to find evidence that for $\alpha \geq \alpha_{c}$, where $\alpha_{c}$ is a critical value which the simulations estimate to be in the interval $1.2-1.3$, the smectic phase is stabilized. This is surprisingly close to the ellipsoidal case. In addition, we use a density-functional approach, based on the Parsons-Lee approximation, to describe smectic and columnar ordering. In combination with a free-volume theory for the crystalline phase, a theoretical phase diagram is predicted. While some qualitative features, such as the enhancement of smectic stability for increasing $\alpha$, and the probable absence of a stable columnar phase, are correct, the precise location of coexistence densities are quantitatively incorrect.
\end{abstract}

PACS numbers: $61.30 . \mathrm{Cz}, 64.70 . \mathrm{mf}$

\section{INTRODUCTION}

Hard interaction models have played an important role in the understanding of the nature and structure of simple liquids and crystals made of particles with spherical symmetry. For anisotropic particles, the hard ellipsoid (HE) and hard-spherocylinder (HSC) models have played a role similar to that of the hard-sphere model (HS) in simple liquids, though these models are not so universal as the HS model. In particular, HE and HSC fluids exhibit an isotropic-nematic phase transition but, while the HSC fluid shows a stable smectic phase, all evidence to date suggests that the HE fluid does not ${ }^{1}$. Clearly, the formation of the smectic phase must be the result of delicate packing effects directly related to particle shape. This feature of the HE fluid is a clear disadvantage in the formulation of perturbation theories for liquid crystals ${ }^{2}$.

The problem of why hard ellipsoids do not get stabilised into a layered smectic structure is in intriguing one. The properties of a perfectly aligned fluid of ellipsoids can be mapped onto those of hard spheres, and the HS fluid does not exhibit phases with order intermediate between the fluid and the crystal. On top of that, orientational freedom probably plays against smectic formation. Recent simulation work on parallel hard ellipsoids augmented by an isotropic square well have shown that, in this system, smectic layers can be stabilised ${ }^{3}$; however, in view of the rather artificial model potential used, the result probably does not reflect any essentially interesting underlying property of ellipsoids.

The physical reason for the absence of smectic order in the HE fluid obviously lies in the geometrical properties of an ellipsoid. Wen and Meyer ${ }^{4}$ addressed the general problem of smectic formation in fluids of parallel hard rods. They provided an explanation in terms of the entropy gain involved in the increased packing efficiency in smectic layers, which more than outweighs the entropy loss associated with the onset of layering with respect to the nematic phase. This efficiency is very much reduced in ellipsoids due to particle shape, since ellipsoids arranged in a layer leave too much void space. Alternatively one may think that increasing packing, which would involve filling this space, entails interlocking between the layers, which promotes crystalline order but discourages formation of the smectic phase.

A question that can be asked to understand this problem from a different perspective is the following: if we perturb the shape of an ellipsoid in the direction of a spherocylinder or a cylinder, both of which exhibit smectic phases ${ }^{5}$, for which particle shape does smectic stability set in? The answer to this question may provide some further insight into the relation between particle geometry and smectic stability. As a bonus, it would help formulate more useful hard-body models that can be used in perturbation theories.

Only a few previous studies have addressed this issue. Of particular relevance to our study is that of Evans ${ }^{6}$, who used an Onsager second-virial coefficient approximation to investigate smectic formation in flu- 
ids made of hard ellipsoids, hard spherocylinders and hard ellipocylinders, a particle with a shape somehow intermediate between that of the first two. The ellipsoids, both parallel and with unconstrained orientations, did not form a smectic phase before the crystal, while the other particles did at some particular density prior to crystallization. It was concluded that ellipsoids are pathological in that they do not form a smectic phase ${ }^{6}$.

In the present paper we again address this problem by studying a continuum of particle shapes, in the limit of parallel particles, but this time interpolating between the ellipsoid and the cylinder by means of a model, the hard superellipsoid (HSE) of revolution, containing an exponent $\alpha$ that can be varied continuously. Monte Carlo (MC) simulations are used to analyse the stability of the smectic phase and other phases with partial positional order (i.e. columnar phase) of the parallel model (PHSE), in the region of geometries close to the ellipsoid, and an approximate phase diagram as a function of $\alpha$ is obtained. The columnar phase is not stable in the phase diagram. MC simulations of freely rotating cut spheres have shown that the columnar phase can be stabilised for some range of aspect ratios ${ }^{7}$. However simulations of parallel cylinders do not give conclusive evidence for the presence of columnar symmetry in the phase diagram 5 , and a recent fundamental measure theory (FMT) for parallel cylinders $\frac{8}{}$ also rules out the columnar phase as a thermodynamically stable phase. However, one of the conclusions of the present paper is that the smectic phase can be stabilised with respect to the nematic phase for some type of PHSE particles. Therefore, in the approximation of parallel particles, ellipsoids do not seem to be pathological; rather it is a continuum of particle shapes, close to the ellipsoidal, that do not exhibit smectic order. To complement these studies, a density-functional theory based on the Parsons-Lee approach ${ }^{9}$ was used to investigate the relative stability of the smectic phase with respect to other phases with lower symmetry. For the crystal phase, a free-volume approximation is used. The main conclusion that can be drawn is that the ParsonsLee approximation, in combination with free-volume theory, only gives a qualitative picture of the relative stability of the phases. The precise location of the transition points are incorrect. Other features, such as the fact that the columnar phase is not stable, appear to be correctly described. Resort to other, more sophisticated theories, e.g. of the FMT type, would be needed to obtain a better description. However, although the initial framework of the FMT for general convex particles was initially developed by Rosenfeld ${ }^{10}$, its application only works for isotropic fluids, as it was shown in Ref. ${ }^{11}$. For some simple geometries (such as parallelepipedic) the FMT can be formulated from first principles with restricted particle orientations ${ }^{12}$. The generalisation of the FMT to geometries like the ones described here seems to be a rather difficult task.

\section{PARTICLE MODEL}

We consider parallel particles with symmetry of revolution (axially symmetric about the $z$ axis). The superellipsoid results by making a superellipse in the $x-z$ plane revolve around the $z$ axis, with the equation

$$
\left(\frac{R}{a}\right)^{2 \alpha}+\left|\frac{z}{b}\right|^{2 \alpha}=1, \quad R=\sqrt{x^{2}+y^{2}}
$$

$a$ and $b$ are the semi-lengths of the particle in the $x y$ plane and along the $z$ axis, respectively, and $\alpha$ is the shape exponent. This equation defines a particle with a geometry interpolating between the ellipsoid $(\alpha=1)$ and the cylinder $(\alpha=\infty)$. Note that the PHSE model is different from that used by Evans ${ }^{6}$, whose model interpolates between an ellipsoid and a spherocylinder. Fig. 1 depicts a few bodies (with $a=b$, which could be called superspheres) for different values of $\alpha$. Thermodynamic and structural properties of such a fluid of parallel particles scale trivially with particle elongation, so that it is sufficient to consider the case $a=b=\sigma_{0} / 2$, with $\sigma_{0}$ the particle breadth.
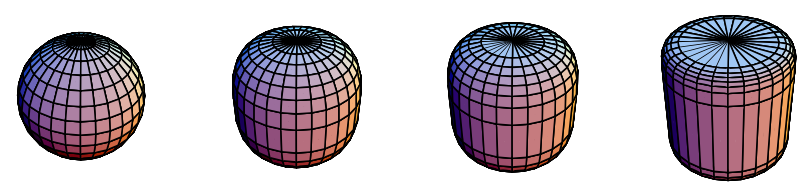

Figure 1: Superellipsoids with $a=b$ (superspheres) for values of $\alpha$ equal to 1, 1.5, 2 and 5 (from left to right).

The stable low-density phase of the PHSE model is a nematic phase (all particles oriented along the $z$ axis and with their centers of mass disordered positionally). At high densities we expect a solid phase with some crystalline structure (the question of which structure is stabilised will be addressed later). At intermediate densities we could, in principle, expect different phases with partial order (smectic or columnar) to get stabilised, depending on the value of $\alpha$.

\section{THEORETICAL TOOLS}

To explore the phase behaviour of the PHSE model we used constant-pressure MC simulation. We have simulated systems of $N=1-2 \times 10^{3}$ particles, with $\alpha=1.0$ 2.0. Both compression runs from the low-density nematic phase and expansion runs from a high-density crystalline structure were conducted. From these, equations of state are obtained, and the different phases may be identified. Relative stability between nematic, smectic and crystal phases (some of which, as we will see, undergo a firstorder phase transition) cannot be ascertained, since no attempt was made at computing absolute free energies. 
Structural quantities, such as density profiles along the ordering direction, $\rho(z)$, and radial distribution functions parallel and perpendicular to this direction, $g_{\|}(z)$ and $g_{\perp}(R)$, were also calculated. All this information allows us to get a picture of the trends in phase behaviour that can be expected as the exponent $\alpha$ is varied.

To complement simulation results, a number of theoretical analyses have been carried out. In the highdensity region, the classical free-volume theory has been implemented. The free-volume free-energy density is written as

$$
\beta \mathcal{F}=-N \log \left(\frac{v_{\text {free }}}{\Lambda^{3}}\right)
$$

where $N$ is the number of particles, $v_{\text {free }}$ the free volume available to a particle when the others are kept fixed in their lattice positions, $\beta=1 / k T$ and $\Lambda$ is the thermal wavelength. For parallel hard cylinders this is an analytical function of density. In the general case this is probably not true, and we have computed $v_{\text {free }}$ by MC integration. In the low-density region, a simple virial expansion for the excess free energy,

$$
\begin{aligned}
\beta \mathcal{F}_{\text {ex }}[\rho]= & -\frac{1}{2} \int d \mathbf{r} \int d \mathbf{r}^{\prime} \rho(\mathbf{r}) \rho\left(\mathbf{r}^{\prime}\right) f\left(\mathbf{r}-\mathbf{r}^{\prime}\right) \\
& -\frac{1}{6} \int d \mathbf{r} \int d \mathbf{r}^{\prime} \int d \mathbf{r}^{\prime \prime} \rho(\mathbf{r}) \rho\left(\mathbf{r}^{\prime}\right) \rho\left(\mathbf{r}^{\prime \prime}\right) \\
& \times f\left(\mathbf{r}-\mathbf{r}^{\prime}\right) f\left(\mathbf{r}^{\prime}-\mathbf{r}^{\prime \prime}\right) f\left(\mathbf{r}-\mathbf{r}^{\prime \prime}\right)+\ldots
\end{aligned}
$$

has been considered to calculate the nematic-smectic bifurcation line $e^{\frac{13}{}}$, with an aim to comparing with the Parson-Lee approach (see later). In the above expansion $f(\mathbf{r})$ is the Mayer function of two parallel PHSE, and $\rho(\mathbf{r})$ the local density distribution. This expansion is meaningful only for the low-density nematic phase and expected to rapidly fail as the system density is increased, but at least it may give an indication as to whether the system is prone to developing smectic ordering and, if so, how this tendency depends on particle shape. Specifically, we have applied a bifurcation analysis based on the above expansion (presented in Appendix A), using second- and third-order terms in $\rho(\mathbf{r})$. At higher density, i.e. in regions where the smectic or columnar phases may be stable, an alternative is to use a Parsons-Lee (PL) scheme ${ }^{9}$. In the PL approach, an approximate resummation of the virial series is performed, using the exact second virial coefficient. That is, we take

$$
B_{n}^{\mathrm{PHSE}}=\frac{B_{n}^{\mathrm{HS}}}{B_{2}^{\mathrm{HS}}} B_{2}^{\mathrm{PHSE}}
$$

where $B_{n}^{\text {HS }}$ are hard-sphere virial coefficients. With this scaling of the PHSE virial coefficients, the excess free energy writes

$$
\beta \mathcal{F}_{\mathrm{ex}}[\rho]=-\frac{\Psi_{\mathrm{HS}}(\eta)}{2 B_{2}^{\mathrm{HS}} \rho_{0}} \int d \mathbf{r} \int d \mathbf{r}^{\prime} \rho(\mathbf{r}) \rho\left(\mathbf{r}^{\prime}\right) f\left(\mathbf{r}-\mathbf{r}^{\prime}\right),
$$

where $\Psi_{\mathrm{HS}}(\eta)$ is the excess free energy per particle of a HS fluid of the same packing fraction $\eta$ as our fluid of PHSE particles. The packing fraction is given by $\eta=\rho_{0} v_{0}$, with $\rho_{0}$ the mean density and $v_{0}$ the particle volume, given by

$$
v_{0}(\alpha)=\frac{\pi \sigma_{0}^{3}}{12 \alpha} B\left(\frac{1}{\alpha}, \frac{1}{2 \alpha}\right),
$$

where $B(x, y)$ is the beta function. For $\Psi_{\mathrm{HS}}(\eta)$ we use the Carnahan-Starling expression, $\Psi_{\mathrm{HS}}(\eta)=(4-3 \eta) \eta /(1-$ $\eta)^{2}$. In the minimisations of the total free energy,

$$
\begin{aligned}
\mathcal{F}[\rho] & =\beta^{-1} \int d \mathbf{r} \rho(\mathbf{r})\left\{\log \left[\rho(\mathbf{r}) \Lambda^{3}\right]-1\right\} \\
& +\mathcal{F}_{\text {ex }}[\rho],
\end{aligned}
$$

where $\mathcal{F}_{\text {ex }}[\rho]$ is given by (3) or (5), the density profile $\rho(\mathbf{r})$ is parametrised in some convenient way. For the smectic and columnar phases at low densities we use a truncated Fourier expansion. For high densities a Gaussian parameterization is used. Details of these calculations are given in Appendix B.

\section{RESULTS}

In this section we use the packing fraction $\eta$ as a convenient measure of density. For a given density, since $v_{0}$ is an increasing function of $\alpha, \eta$ increases slightly from the ellipsoid to the cylinder. In Fig. 2, MC data for reduced presure $p v_{0} / k T$ versus packing fraction $\eta$, for all the particle shapes considered, are shown (from now on we only show simulation results for the systems with $N \simeq 10^{3}$ particles; selected checks with twice as many particles did not give any quantitative differences). In all cases the low-density phase is a nematic (N phase), since all particles are parallel, whereas the system crystallises at high density ( $\mathrm{K}$ phase). We begin by discussing the high-density region, where the crystal phase is the stable phase.

As expected, compression runs starting from the lowdensity nematic phase (open squares in Fig. 2) ultimately create a defected crystal, via a first-order phase transition (this appears as a clear density discontinuity in all cases). Expansion runs from a perfect high-density crystal (filled squares in Fig. 22) give a solid branch with defect-free structures in most instances. However, in small systems defects consisting of columns of particles that have moved along the director by a fraction of the unit cell are created. This may give the impression that averaged structures correspond to a stable columnar phase. A related situation was found in the simulations of Veerman and Frenkel on parallel hard cylinders ${ }^{5}$, where a strong dependence of columnar stability on system size was found.

To investigate this, we prepared initial configurations with columnar symmetry at high density; in expansion runs, the system always stayed as a columnar phase (triangles in Fig. 21). Therefore, for large system sizes, systems with columnar and crystalline structures look 

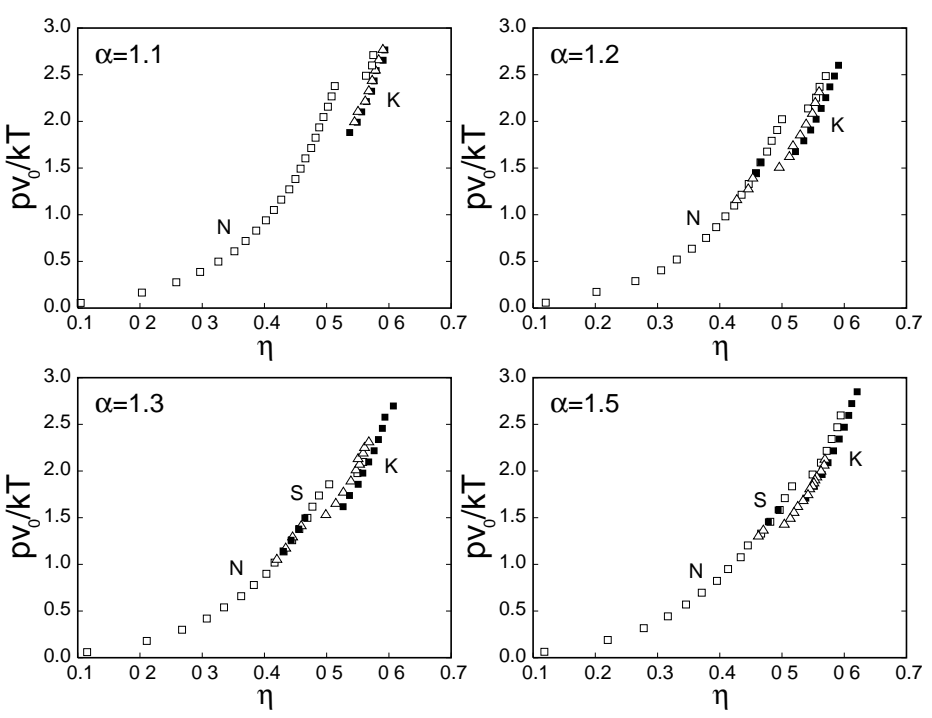

Figure 2: Equation of state pressure-packing fraction for various values of shape parameter $\alpha$ (shown in each panel), as obtained from constant-pressure Monte Carlo simulations. Open squares: compression runs starting from nematic phase. Filled squares: expansion runs starting from crystalline phase with ABC symmetry. Triangles: expansion runs starting from columnar symmetry. Identification of phases is made with symbols (N, S and $\mathrm{K}$ for nematic, smectic and crystal, respectively).

as though they are actually mechanically stable, and there seems to be a large free-energy barrier between the two structures. However, compressions from the nematic never give rise to configurations containing partial columnar order (which would seem easier to generate than full three-dimensional order). Without explicit calculation of free energies, no definite conclusion can be reached on the relative stabilities of columnar and crystalline phases; however, given that no clear evidence for columnar ordering has been found in simulations of parallel hard cylinders ${ }^{5}$, and that FMT calculations on the same system predict that columnar order is not thermodynamically stable ${ }^{8}$, we believe it unlikely that a columnar phase may get stabilised in PHSE.

As the crystal is expanded to lower density the system looses translational order (either totally or partially), and becomes fluid via a discontinuity in density. Fig. 3) (c) shows the radial distribution functions along and perpendicular to the director, $g_{\|}(z)$ and $g_{\perp}(R)$, in the crystal phase for the case $\alpha=1.5$. Both present a high degree of structure, as expected in a crystalline phase. Therefore all evidence suggests that there is a first-order fluid-crystal (i.e. freezing) transition.

The question on the nature of the crystalline phase and how the system is prepared in the expansion runs deserves some comments. In fact the symmetry of the structure seems to change at some value of $\alpha$. The generic crystal structure consists of stacked triangular layers, either in phase (AAA structure) or out of phase. The latter

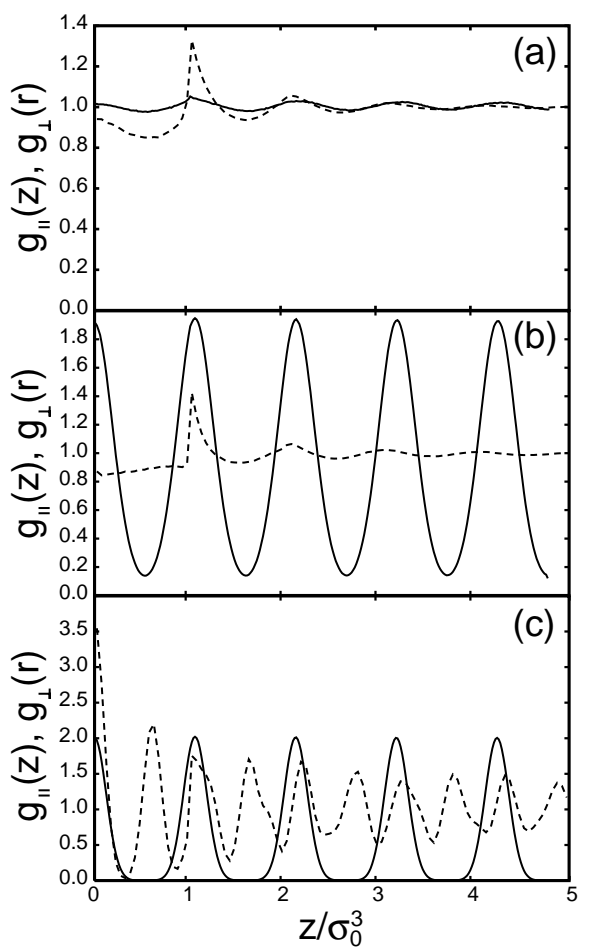

Figure 3: Radial distribution functions along (continuous line) and perpendicular to (dashed line) the director, $g_{\|}(z)$ and $g_{\perp}(R)$, for the PHSE fluid with $\alpha=1.5$; (a) $\eta=0.38$; (b) $\eta=0.43$; and (c) $\eta=0.53$.

may have $\mathrm{ABCA} . .$, ABAB..., or random-stacking structures, all of these being compact structures (i.e. they share the same value of the close-packing density). A priori one could consider the AAA, ABC and ABAB structures to be the most stable candidates, reflecting simple hexagonal-, face-centred-cubic- and hexagonal-closepacked-like symmetries, respectively (the final structures obtained by the simulations do not have these exact symmetries, since the intralayer unit-cell distance does not exactly correspond to the interlayer spacing expected in these structures; obviously this is a consequence of the asymmetry of the particles along and perpendicular to their symmetry axes). The situation, for lack of a more detailed analysis based on free-energy estimations is, as usual, uncertain (cf. the old debate on the hard-sphere crystal $^{14}$ ), presumably due to very small free-energy differences. For freely rotating ellipsoids a recent work has shown the existence of a different crystal packing consisting of a simple monoclinic lattice with a basis of two ellipsoids with different orientations ${ }^{15}$ (of course this structure cannot occur in our parallel-particle model). In our case of parallel PHSE particles with $\alpha \leq 1.5$, simulations with between 8 to 12 layers along the $z$ direction may give structures with any type of stacking depending on how the initial configuration is prepared (in the limit $\alpha=1$, we know that ABC stacking is preferred $\left.{ }^{14}\right)$. However, in the limit $\alpha \rightarrow \infty$, the AAA structure is clearly more stable, and it is expected that somewhere in the 
interval $1.5<\alpha<\infty$ there is a change in the nature of the crystal phase. Some preliminary simulations indicate that the critical value may be between 1.5 and 2.0 (e.g. a crystal with $\alpha=1.5$, when prepared with AAA stacking, shows a strong tendency to evolve towards random stacking, whereas the system with $\alpha=2.0$, when prepared with ABC stacking, evolves towards AAA stacking), but free-volume theory gives a rather higher value (see later).

Now the central question of the present paper is whether there exists an intermediate, stable fluid phase between the low-density nematic and the high-density crystal phase. In the limit $\alpha=1$ (parallel ellipsoids) ones knows for certain that there are no phases with partial (smectic or columnar) order $\stackrel{1}{*}$. By contrast, in the opposite limit $\alpha=\infty$ (parallel cylinders) previous MC simulations ${ }^{-5}$ indicate that the nematic phase changes to a smectic phase via a continuous transition. Clearly in our interaction model, which interpolates between these two limits, there must be a value of $\alpha$ beyond which the smectic phase becomes stable. Actually this is the case. In Fig. 2 cases where a smectic phase has been identified are indicated by a corresponding label (S for smectic). Evidence for the $\mathrm{S}$ phase comes from density distributions (see later), as the pressure shows no sign of a N-S transition, pointing to a continuous transition. We should note that recent simulations on freely-rotating hard spherocylinders in the limit of infinite aspect ratio have shown that the nematic-smectic transition is of first order ${ }^{16}$. Thus, we can conclude that the parallel alignment constraint is responsible for the second-order nature of the N-S transition of hard cylinders, and this could also be the case in our model. By increasing the pressure further (compression run), the smectic phase transforms into a defected crystal phase via a first-order phase transition.

As mentioned above, our simulations indicate that a smectic phase stabilises for finite $\alpha$, i.e. in the range $\alpha_{c} \leq \alpha<\infty$, where $\alpha_{c}$ is some critical value. Indirect evidence comes from computation of density profiles, smectic order parameter (not shown) and correlation functions. In Fig. 4 the evolution of the density profile $\rho(z)$, as density is increased, is shown for the case $\alpha=1.5$. The onset of smectic order is clearly identified by the smooth appearance of density peaks. The density profiles exhibit a clear stratification at a packing fraction $\eta_{\mathrm{NS}} \simeq 0.43$. That the high-density phase in question is smectic, and not crystalline, can be concluded from the radial distribution functions along and perpendicular to the director, shown in Figs. 3(a) and (b), which point to in-plane fluid-like correlations in the intermediate phase.

Analysis of the case $\alpha=1.2$, in contrast, suggests no evidence for a smectic phase: the nematic fluid freezes directly into a crystal (see Fig. 2), similarly to the case of ellipsoids. The intermediate case $\alpha=1.3$, however, does show signs of smectic stability. > From all the information collected for the various systems analysed, we estimate the critical value of $\alpha$ associated with the onset of stability of the smectic phase to be in the range

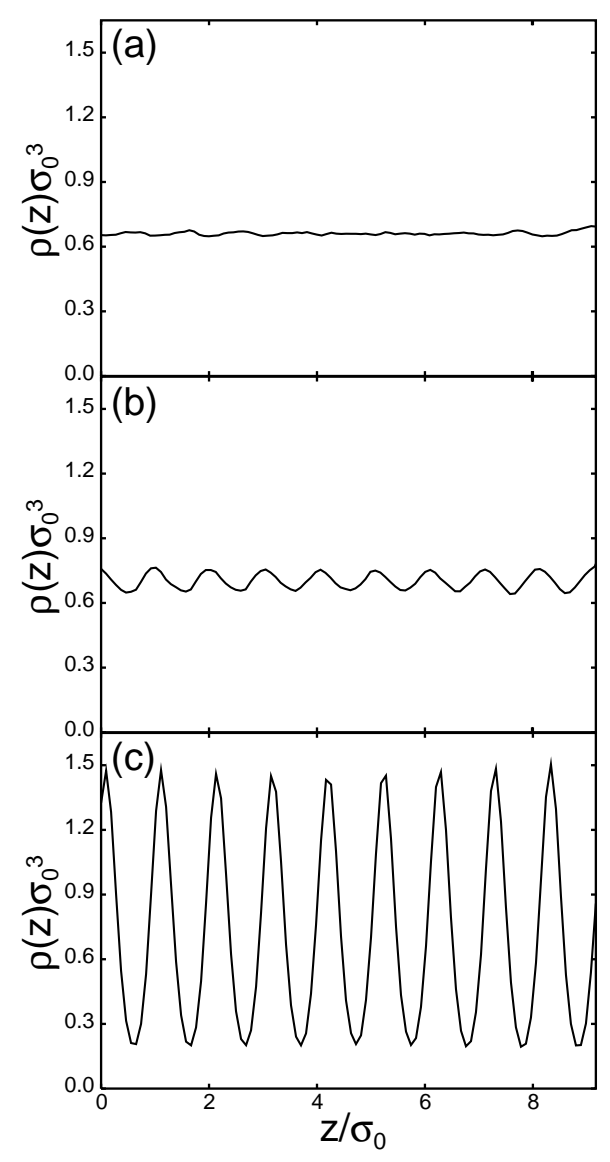

Figure 4: Density profiles $\rho(z)$ for the PHSE fluid with $\alpha=$ 1.5 ; (a) $\eta=0.39$; (b) $\eta=0.43$; and (c) $\eta=0.45$.

$\alpha_{c}=1.2-1.3$.

Fig. 5 summarises our results in the form of a phase diagram of packing fraction $\eta$ vs. inverse exponent $\alpha^{-1}$. For $\alpha \gtrsim 1.2$ the smectic phase ( $\mathrm{S}$ in the graph) is stable. Simulation data for the limit $\alpha=\infty$ (cylinders, filled triangles and squares) are taken from simulations by Veerman and Frenkel ${ }^{5}$, and are essentially exact as they were inferred from simulations incorporating freeenergy calculations. Data for hard spheres (filled circles) are taken from Hoover and Ree $\frac{17}{7}$. In the other cases transition densities for the nematic-smectic, nematiccrystal and smectic-crystal transitions are only approximate. As already mentioned, the first (indicated by open circles) is of second order. The others (open triangles and squares) are of first-order, with a wide density gap; since no free energies were computed, we only plot, using vertical bars, approximate limits of metastability of the two phases involved (the symbols are only the average packing-fraction values inferred from the estimated limits of metastability).

A second aim of our study is to rationalise the findings from computer simulation using theoretical models. As mentioned in Sec. III, we have considered three theoretical models: a low-density virial expansion up to third or- 


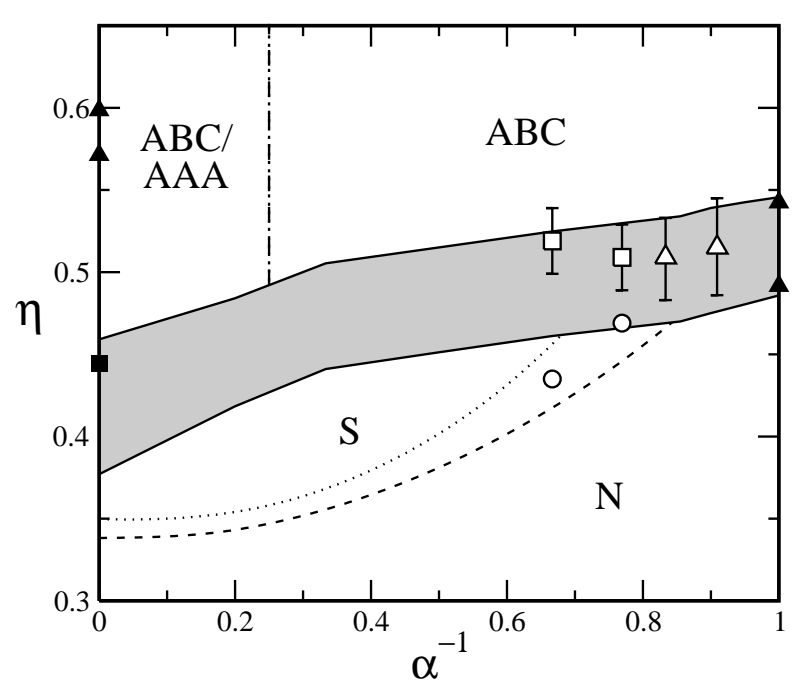

Figure 5: Phase diagram in the plane packing fraction $\eta-$ inverse shape parameter $\alpha^{-1}$. Continuous lines: coexistence boundaries for the smectic-solid transition from PL theory for the smectic and FV theory for the solid; shaded region: two-phase region from previous approximations; dashed line: nematic-smectic spinodal from PL theory; dotted: nematicsmectic spinodal from V3 theory; filled triangles: simulation results for coexistence packing fractions of smecticsolid transition in parallel cylinders $(\alpha=\infty)$ from Veerman and Frenkel ${ }^{5}$, and for liquid-solid coexistence in hard spheres $^{17}(\alpha=1)$; filled square: nematic-smectic spinodal from computer simulation of Veerman and Frenke ${ }^{15}$; open circles: our simulation estimates for nematic-smectic spinodal; open squares: our simulation estimates for first-order smectic-solid transition; open triangles: our simulation estimates for first-order nematic-solid transition. In the latter two cases the vertical bars only indicate limits of metastability. Labels indicate stable phases; N, nematic; S, smectic; ABC and AAA, crystalline solids with corresponding symmetries. Vertical dot-dashed line: approximate limit of degeneracy of $\mathrm{ABC}$ and AAA structures within FV theory.

der in density for the low-density nematic phase (V3), a resummed virial expansion of the Parsons-Lee type (PL) for intermediate densities, and free-volume (FV) theory for the high-density crystal. The V3 theory was initially used to analyse possible bifurcations of the nematic phase to smectic or columnar phases $\frac{13}{}$. The qualitative results as far as the continuous nematic-smectic transition is concerned are the same for both V3 and PL theories; they only differ quantitatively, as can be seen in Fig. 5 . For large values of $\alpha^{-1}$ (the only available from simulations), the MC data are bracketed by the two theories, but the trend that the packing fraction at bifurcation increases with $\alpha^{-1}$ in this regime is captured correctly. This increase is basically due to the decrease in particle volume as cylinders change to ellipsoids. The differences between the V3 and PL predictions can be traced back to their different treatment of correlations: V3 includes three-body correlations exactly, but the remaining terms are neglected, while PL only includes the exact two-body correlations but approximately resums the higher-order density correlations.

The PL theory, together with the FV theory for the crystal, were used to compute the nematic-solid and smectic-solid transitions. These are first-order transitions, with a wide density gap. The two-phase region merges with the nematic-smectic spinodal coming from lower densities at a critical end-point located at $\alpha_{c} \simeq 1.2$; this value approximately agrees with that inferred from the simulations. For lower values of $\alpha$ there is direct coexistence between nematic and solid phases, and the theory also seems to agree with simulations in the limit of hard spheres (this agreement is fortuitous as it is well known that the PL theory is inappropriate to model spatial correlations in the hard-sphere fluid; the weighted-density theory $\underline{18}$ or a theory based on FMT $\underline{19}$ are known to be more adequate). In the opposite limit (cylinders), however, there is a big discrepancy with simulation, not only in the location of the nematic-smectic spinodal and the smectic-solid phase transition, but also in the density gap of the latter which is significantly overestimated as compared to the MC simulations. In this limit the weighteddensity type theories, developed for a $\mathrm{HSC}$ fluid ${ }^{20}$, are obviously more appropriate to predict the spatial correlations. The PL and FV theories were also used to compute the smectic-columnar and columnar-solid transitions. These results are not plotted in the phase diagram since the region of columnar stability is always preempted by crystallization directly from the smectic phase; this feature also agrees with simulations which, as mentioned before, do not seem to give conclusive evidence for stable columnar ordering in this model.

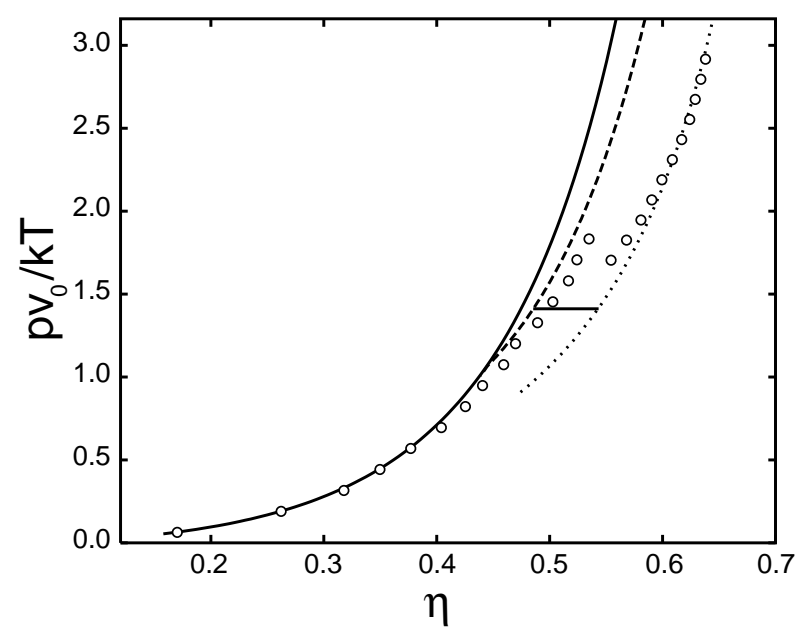

Figure 6: Equation of state for the PHSE system with $\alpha=$ 1.5. Symbols: MC results; continuous line: nematic branch as obtained from CS approximation; dashed line: smectic branch from PL theory; dotted line: solid branch according to $\mathrm{FV}$ theory.

The quality of the different approximations, in the density range where each of them was used, can be checked by examining the equation of state (EOS). This is done 
in Fig. 6, which refers to the case $\alpha=1.5$. The figure also contains the MC simulation data. The PL theory, which reduces to the Carnahan-Starling approximation for the nematic phase (continuous line), represents correctly the EOS up to packing fractions of $\eta \simeq 0.4$, just before the transition to the smectic phase occurs $\left(\eta_{\mathrm{NS}} \simeq 0.43\right)$. The PL theory is not as accurate for the smectic phase (dashed line), since it overestimates the pressure. The nematic-smectic transition density (bifurcation point) is reasonably well reproduced. In the solid branch (dotted line), the equation of state is accurately represented (in fact better as the density approaches the close-packing limit), as expected, but the smectic-solid transition is not correctly reproduced (value of pressure, location of transition densities and density gap), due to the defects in the smectic and solid equations of state.

\section{CONCLUSIONS}

We can conclude from our study that there is clear evidence that in the PHSE fluid smecticity begins to be favoured entropically beyond a critical value of the exponent $\alpha_{c} \simeq 1.2-1.3$; this is surprisingly close to the value corresponding to ellipsoids $(\alpha=1)$. Therefore, the fluid of parallel hard ellipsoids does not seem to be pathological or special in not exhibiting a stable smectic phase; rather, there is a family of particle shapes beyond the ellipsoid that do not possess stable smectic phases, although the extent of the family in parameter space is small.

The explanation for this behaviour lies, of course, in the packing efficiency of these hard-particle fluids. The formation of the smectic phase is the result of a delicate packing effect directly related to particle shape. In the HE fluid, the ellipsoidal geometry creates a large tendency for particles to interlock at their ends, which favors the stabilisation of the crystal phase with detriment to the smectic phase. This effect is not relevant in the case of HSC and sets in at some intermediate particle shape.

Our simulation results seem to indicate that the columnar phase is not stable for this family of parallel rods. But free-energy computations will be needed to settle this question. However, in common with the case of parallel cylinders, it is not likely that the columnar phase will be stable in our model. All these conclusions correspond to the model of parallel particles. Analysis should be extended to consider particles with free orientational degrees of freedom. Given the high degree of orientational order of the smectic phases, we do no think the conclusions drawn from our study will be changed substantially.

As a byproduct of our analysis, we have assessed the validity of various theoretical approximations by comparison with the simulation results. A combination of freevolume and PL theories qualitatively predicts the correct phase stability, but the agreement is far from quantitative, except in the parameter region close to the spheres; in particular, the value of shape anisotropy beyond which smectic stability sets in is quite in agreement with simulations. Use of the PL theory to consistently describe all phases is not adequate, since this theory progressively degrades as order builds up in the system: therefore, the agreement is probably fortuitous.

An obvious avenue to improve the theoretical treatment is to use a more sophisticated theory, which is still to be formulated. A promising approach is FMT, which one hopes would correctly predict the relative stability of non-uniform phases; this belief is based on FMT calculations applied to the particular case of parallel hard cylinders, as shown in Ref. $\stackrel{8}{*}$. However, the extension of this theory to superellipsoidal geometry would demand the use of strong approximations, in the line of Ref ${ }^{21}$ where only the first terms of the excess free-energy asymptotic expansion with respect to $\kappa^{-1}$ (with $\kappa$ the particle aspect ratio) are taken into account. This procedure could disrupt the high predictive power of FMT as concerns the precise location of phase transitions and the relative stability of different non-uniform phases. Further investigation of this problem would be needed but is left for future work.

Interesting questions on the effect of particle shape on the depletion interaction between two anisotropic bodies mediated by small spherical particles can be studied within the present model. Recent studies have shown that particle geometry has a strong effect on the depletion forces ${ }^{22}$. The present model can be used to tune the geometry of the particle with a single parameter, from ellipsoids to cylinders, and allows the study of the evolution of the depletion potential with respect to $\alpha$ when two superellipsoids are immersed in a sea of small hard spheres. Work in this direction is in progress.

\section{Acknowledgments}

Y.M.-R. gratefully acknowledges financial support from Ministerio de Educación y Ciencia (Spain) under a Ramón y Cajal research contract and the MOSAICO grant. This work is part of the research projects Nos. FIS2005-05243-C02-01 and FIS2007-65869-C03-01, also from Ministerio de Educación y Ciencia, and grant No. S-0505/ESP-0299 from Comunidad Autónoma de Madrid (Spain).

\section{Appendix A: SPINODAL INSTABILITY OF N-S TRANSITION}

The spinodal instability coincides with the location of the continuous N-S phase transition. The instability condition can be calculated by solving the following set of equations

$$
1-\rho \hat{c}(\eta, q)=0, \quad \frac{\partial \hat{c}(\eta, q)}{\partial q}=0
$$


where $\hat{c}(\eta, q)$ is the Fourier transform of the direct correlation function. The equations are to be solved for $\eta^{*}$ and $q^{*}$, which are the value of the packing fraction and the wave number of the smectic phase, both at bifurcation. The second equation refers to the absolute minimum of $-\hat{c}(\eta, q)$ as this is a strongly oscillatory function of $q$.

To calculate the spinodal curve a model for the direct correlation function is needed. The third virial approach, Eqn. (3), gives

$$
\hat{c}(\eta, q)=\hat{f}(q)+\rho \Delta(q),
$$

where the Fourier transform of the Mayer function $\hat{f}(q)=$ $\int d \mathbf{r} e^{i q z} f(\mathbf{r})$ gives the following result for a PHSE particle with unit breadth and height:

$$
-\hat{f}(q)=\frac{4 \pi}{q} \int_{0}^{1} d z z \sin \left[q\left(1-z^{2 \alpha}\right)^{1 /(2 \alpha)}\right]
$$

and

$$
\begin{aligned}
-\Delta(q) & =\int d \mathbf{r} e^{i q z} f(\mathbf{r}) \Delta V(\mathbf{r}), \\
\Delta V(\mathbf{r}) & =\int d \mathbf{r}^{\prime} f\left(\mathbf{r}^{\prime}\right) f\left(\mathbf{r}-\mathbf{r}^{\prime}\right) .
\end{aligned}
$$

For each value of $q$ (which is understood to be given in units of $\sigma_{0}$ ), taken from a set of equally-spaced points, the function $\Delta(q)$ was calculated by MC integration for a fixed $\alpha$. Then the equations (A1) were solved to find the spinodal curve $\eta^{*}(\alpha)$. Finally, the Parsons-Lee approach gives $\hat{c}(\eta, q)=\frac{1}{4} \Psi_{\mathrm{HS}}(\eta) \hat{f}(q)$.

\section{Appendix B: PARAMETERIZATIONS OF THE PARSONS-LEE THEORY}

The density distributions for smectic and columnar symmetries were parameterized using two different approaches. The first, based on a Fourier expansion, is more adequate for relatively low mean densities, while the other, a parameterization based on two parameters (one being a measure of the width of the density peaks, the other being the smectic or columnar lattice parameters periods), is more appropriate for high-density phases, as the numerical convergence of the minimization schemes is much easier when the parameter space is reduced to two variables. We have used the following parameterization for the density profile of the smectic phase:

$$
\rho(z)=\frac{\rho_{0}}{I_{0}(\lambda)} \exp (\lambda \cos q z)
$$

where $\rho_{0}=d^{-1} \int_{0}^{d} d z \rho(z)$ is the mean density, $q=2 \pi / d$ the wave number ( $d$ being the smectic period), $\lambda$ the minimization parameter, and $I_{0}(\lambda)$ the zeroth order modified Bessel function. Inserting this expression into the Parsons-Lee functional, Eqn. (5), we find the following expression for $\varphi_{\mathrm{ex}}=\beta \mathcal{F}_{\mathrm{ex}} / N$, the excess part of the freeenergy per particle and unit thermal energy:

$\varphi_{\mathrm{ex}}=-\frac{\Psi_{\mathrm{HS}}(\eta)}{2 B_{2}^{\mathrm{HS}} \rho_{0}^{2}} d^{-1} \int_{0}^{d} d z \rho(z) \int_{-\infty}^{\infty} d z^{\prime} \rho\left(z^{\prime}\right) \tilde{f}\left(z-z^{\prime}\right)$,

where $B_{2}^{\mathrm{HS}}=4 v_{0}(\alpha)$ (since the volume of the reference HS particle is made to coincide with that of the PHSE). Also we have defined $\tilde{f}(z)=\int d \mathbf{r}_{\perp} f\left(\mathbf{r}_{\perp}\right)$ as the integrated Mayer function over the transverse area, with $\mathbf{r}_{\perp}=(x, y)$. Note that the HS free-energy per particle is $\Psi_{\mathrm{HS}}(\eta)=(4-3 \eta) \eta /(1-\eta)^{2}$. By calculating the integral involved in Eq. (B2) we find explicitly

$$
\varphi_{\mathrm{ex}}=2 \Psi_{\mathrm{HS}}(\eta) C_{\alpha} \chi(\lambda ; \alpha),
$$

where

$$
\begin{aligned}
C_{\alpha} & =\frac{3 \alpha}{2} B^{-1}\left(\frac{1}{\alpha}, \frac{1}{2 \alpha}\right), \\
\chi(\lambda ; \alpha) & =I_{0}^{-2}(\lambda) \int_{0}^{1} d z\left(1-z^{2 \alpha}\right)^{1 / \alpha} I_{0}\left(2 \lambda \cos \frac{q^{*} z}{2}\right),
\end{aligned}
$$

and $q^{*}=2 \pi \sigma_{0} / d$. Finally, the ideal part of the freeenergy per particle and unit thermal energy, $\varphi_{\mathrm{id}} \equiv$ $\beta \mathcal{F}_{\text {id }} / N$, can be found as

$$
\varphi_{\mathrm{id}}=\ln \eta-1+\lambda \frac{I_{1}(\lambda)}{I_{0}(\lambda)}-\ln I_{0}(\lambda),
$$

where $I_{1}(x)$ is the first order modified Bessel function. We have minimized the total energy $\varphi=\varphi_{\mathrm{id}}+\varphi_{\mathrm{ex}}$ with respect to $\lambda$ and $d$ for a fixed value of $\eta$.

For the columnar phase, the parameterization chosen is a sum of Gaussian peaks centered at the sites of the triangular lattice:

$$
\rho\left(\mathbf{r}_{\perp}\right)=\frac{\lambda_{0} \rho_{0} A_{\mathrm{cell}}}{\pi} \sum_{\mathbf{k}} \exp \left[-\lambda_{0}\left(\mathbf{r}_{\perp}-\mathbf{R}_{\mathbf{k}}\right)^{2}\right],
$$

and normalized in such a way that integration over the unit cell of area $A_{\text {cell }}=\sqrt{3} a^{2} / 2$ ( $a$ being the lattice parameter of the triangular cell) gives the mean density $\rho_{0}$. The position of the lattice sites are $\mathbf{R}_{\mathbf{k}}=k_{1} \mathbf{a}_{1}+k_{2} \mathbf{a}_{2}$ $\left(k_{i} \in Z\right)$ with $\mathbf{a}_{n}=\frac{a}{2}\left(\sqrt{3},(-1)^{n}\right)$ being the vectors of the triangular lattice. The Gaussian width is controlled by the parameter $\lambda_{0}$ which, together with $a$, define the set of minimization variables. The excess part of the free-energy per particle can be calculated as

$$
\begin{aligned}
\varphi_{\mathrm{ex}} & =\frac{\gamma}{\pi} A_{\mathrm{cell}}^{*} \Psi_{\mathrm{HS}}(\eta) C_{\alpha} \sum_{\mathbf{k}} e^{-\frac{\gamma}{2}\left(R_{\mathbf{k}}^{*}\right)^{2}} \\
& \times \int_{0}^{1 / a^{*}} d r r\left[1-\left(r a^{*}\right)^{2 \alpha}\right]^{1 /(2 \alpha)} e^{-\frac{\gamma}{2} r^{2}} I_{0}\left(\gamma R_{\mathbf{k}}^{*} r\right),
\end{aligned}
$$


where $\gamma=\lambda_{0} a^{2}, a^{*}=a / \sigma_{0}, R_{\mathbf{k}}^{*}=\left|\mathbf{R}_{\mathbf{k}}\right| / a$, and $A_{\text {cell }}^{*}=\frac{\sqrt{3}}{2}\left(a^{*}\right)^{2}$ (the unit cell area in dimensionless units). Correspondingly the ideal part of the free-energy per particle is defined by

$$
\begin{aligned}
\varphi_{\mathrm{id}} & =\ln [\sqrt{3} \eta \gamma /(2 \pi)]-1+\frac{1}{\pi} \int_{0}^{2 \pi} d \phi \int_{0}^{\infty} d r r \\
& \times e^{-r^{2}} \ln \left[\sum_{\mathbf{k}} e^{-\left(\mathbf{r}-\sqrt{\gamma} \mathbf{R}_{\mathbf{k}}^{*}\right)^{2}}\right]
\end{aligned}
$$

An useful approximation for the ideal part when $\gamma \gg 1$ can be obtained by taking $\mathbf{R}_{\mathbf{k}}^{*} \rightarrow \mathbf{0}$ in Eqn. (B9), with the result

$$
\varphi_{\mathrm{id}}=\ln [\sqrt{3} \eta \gamma /(2 \pi)]-2 .
$$

Also, the excess part (B8) can be approximated by taking only the terms $\mathbf{k}=(0, \pm 1), \mathbf{k}=( \pm 1,0), \mathbf{k}=(1,-1)$ and $\mathbf{k}=(-1,1)$ in the sum (i.e. considering only the nearest neighbours of a given site).

Now we proceed to describe the other parametrization used for minimization at low densities: a truncated Fourier expansion of the density the density profile. This expansion for the smectic symmetry is

$$
\rho(z)=\rho_{0} \psi(z)=\rho_{0}\left[1+\sum_{n>0}^{N} \rho_{n} \cos (q n z)\right],
$$

with $\rho_{n}$ the Fourier amplitudes. Using this parameterization, the excess part of the free-energy per particle reads

$$
\varphi_{\mathrm{ex}}=\Psi_{\mathrm{HS}}(\eta) \sum_{n \geq 0}^{N} \theta_{n} T_{n} \rho_{n}^{2},
$$

where $\theta_{n}=\left(1+\delta_{n, 0}\right) / 2$ and

$$
T_{n}=\frac{4 C_{\alpha}}{q_{n}} \int_{0}^{1} d z z \sin \left[q_{n}\left(1-z^{2 \alpha}\right)^{1 /(2 \alpha)}\right],
$$

with $q_{n}=q \sigma_{0} n$, while the ideal part is

$$
\varphi_{\mathrm{id}}=\ln \eta-1+2 \int_{0}^{1 / 2} d z \psi(d z) \ln \psi(d z) .
$$

For the columnar symmetry the Fourier expansion of the density distribution reads

$$
\begin{aligned}
\rho\left(\mathbf{r}_{\perp}\right) & =\rho_{0} \psi\left(\mathbf{r}_{\perp}\right)=\rho_{0}\left[1+\sum_{n_{1}, n_{2}>0}^{N_{1}, N_{2}} \rho_{n_{1} n_{2}}\right. \\
& \left.\times \cos \left(\frac{2 \pi n_{1} x}{a \sqrt{3}}\right) \cos \left(\frac{2 \pi n_{2} y}{a}\right)\right],
\end{aligned}
$$

where $\rho_{n_{1} n_{2}}$ are the two-dimensional Fourier amplitudes. After insertion of Eqn. (B15) into the Parsons-Lee expression for the excess free energy, we obtain

$$
\varphi_{\mathrm{ex}}=\Psi_{\mathrm{HS}}(\eta) \sum_{n 1, n 2 \geq 0}^{N_{1}, N_{2}} \theta_{n_{1} n_{2}} T_{n_{1} n_{2}} \rho_{n_{1} n_{2}}^{2},
$$

for the excess free-energy per particle, where $\theta_{n_{1} n_{2}}=$ $\left(\delta_{n_{1}+n_{2}, 0}+\delta_{n_{1}, 0}+\delta_{n_{2}, 0}+1\right) / 4$, and

$$
T_{n_{1} n_{2}}=4 C_{\alpha} \int_{0}^{1} d z z\left(1-z^{2 \alpha}\right)^{1 /(2 \alpha)} J_{0}\left(q_{n_{1} n_{2}} z\right),
$$

with $J_{0}(x)$ the zeroth order Bessel function and $q_{n_{1} n_{2}}=\frac{2 \pi}{a^{*}} \sqrt{\frac{n_{1}^{2}}{3}+n_{2}^{2}}$. The ideal part of the free-energy per particle for columnar symmetry can be calculated as

$$
\begin{aligned}
\varphi_{\mathrm{id}} & =\ln \eta-1+\frac{8}{3 \sqrt{3} a^{2}} \int_{0}^{\sqrt{3} a / 2} d x \int_{0}^{a-x / \sqrt{3}} d y \\
& \times \psi(x, y) \ln \psi(x, y) .
\end{aligned}
$$

This time the total free-energy per particle should be minimized with respect to the lattice parameter $a$ and also with respect to the Fourier amplitudes $\rho_{n_{1} n_{2}}$. While the Fourier parameterizations were used for the minimization of the total free-energy of the smectic and columnar phases at low densities (up to $\eta \sim 0.55$ ), the corresponding parameterizations using only two parameters, described above, were used to minimize the free energies per particle of these phases at higher densities. Both approaches were checked for consistency at intermediate densities.
* Electronic address: yuri@math.uc3m.es

$\dagger$ Electronic address: enrique.velasco@uam.es URL: http://www .uam.es/enrique.velasco

1 D. Frenkel, Mol. Phys. 60, 1 (1987).

2 L. Mederos and D. E. Sullivan, Phys. Rev. A 39, 854 (1989).

3 E. de Miguel and E. Martín del Río, Phys. Rev. Lett. 95, 217802 (2005).

${ }^{4}$ X. Wen and R. B. Meyer, Phys. Rev. Lett. 59, 1325 (1987).
5 J. A. C. Veerman and D. Frenkel, Phys. Rev. A 43; 4334 (1991).

${ }^{6}$ G. T. Evans, Mol. Phys. 76, 1359 (1992).

7 J. A. C. Veerman and D. Frenkel, Phys. Rev. A 45, 5632 (1991); M. A. Bates and D. Frenkel, Phys. Rev. E 57, 4824 (1998).

8 J. A. Capitán, Y. Martínez-Ratón and J. A. Cuesta (unpublished).

9 J. D. Parsons, Phys. Rev. A A19, 1225 (1979); S. D. Lee, 
J. Chem. Phys. 87, 4972 (1987); 89, 7036 (1988).

10 Y. Rosenfeld, Phys. Rev. E 50, R3318 (1994).

11 A. Chamoux and A. Perera, J. Chem. Phys. 104, 1493 (1996).

12 J. A. Cuesta and Y. Martínez-Ratón, Phys. Rev. Lett. 78, 3681 (1997); J. Chem. Phys. 107, 6379 (1997).

13 B. Mulder, Phys. Rev. A 35, 3095 (1987).

14 D. Frenkel and A. J. C. Ladd, J. Chem. Phys. 81, 3188 (1984); L. V. Woodcock, Nature 385, 141 (1997); P. G. Bolhuis, D. Frenkel, S.-C. Mau and D. A. Huse, Nature 388, 235 (1997); L. V. Woodcock, Nature 388, 236 (1997).

15 P. Pfleiderer and T. Schilling, Phys. Rev. E 75, 020402(R) (2007).
16 J. M. Polson and D. Frenkel, Phys. Rev. E 56, R6260 (1997).

17 W. G. Hoover and F. H. Ree, J. Chem. Phys. 49, 3602 (1968).

18 P. Tarazona, Phys. Rev. A 31, 2672 (1985).

19 P. Tarazona, Phys. Rev. Lett. 84, 694 (2002).

20 A. M. Somoza and P. Tarazona, Phys. Rev. A 41, 965 (1990).

21 A. Esztermann and M. Schmidt, Phys. Rev. E 70, 022591 (2004).

22 Y. Martínez-Ratón, G. Cinacchi, E. Velasco, and L. Mederos, Eur. Phys. J. E 21, 175 (2006). 